\title{
Cartografiar la formación
}

\author{
Maria Graciela DI FRANCO
}

$\mathrm{L}$ a Ley No 26.206 Ley de Educación Nacional generó numerosos y sustanciales cambios en las políticas públicas de nuestro país. A riesgo de abusar de la simplificación, y sólo para invitar a un debate hacemos referencia a dos en vinculación a la temática que nos convoca: escolaridad secundaria obligatoria y la formación profesional de quienes ejercen la docencia.

Del primer caso, quienes se especializan en análisis institucional nos han señalado lo que implica la mudanza del mandato fundacional de una profesión nacida para formar estudiantes que iban al secundario "porque querían" -dado que el carácter obligatorio quedaba restringido a la primaria-, a pensarse docentes de una escuela secundaria obligatoria. Este cambio implica repensar las instituciones formadoras en el sentido de su proyecto político.

Comienzan a pensarse cambios en la formación de los profesorados universitarios. Es así como la Facultad de Ciencias Humanas de la UNLPam inicia sus discusiones a fin de modificar sus planes de estudio. La formación de los/as estudiantes del profesorado parece requerir la modificación de un currículum cuya finalidad es el estudio de las diferentes disciplinas, para pensar un currículum que oferte posibilidades de activar las capacidades de actuación docente en situaciones reales, complejas, inciertas y problemáticas que requieran saber disciplinar y pedagógico articulados y que generen conocimiento profesional del profesor.

En el año 2009 se aprueban los nuevos planes con la prescripción del Campo de la Práctica (génesis que hemos descripto en números anteriores). Estos debates institucionales se replicaron en otras universidades. En 2012 diversos documentos de la Comisión Mixta ANFHE-CUCEN dieron origen a la formulación de los "Lineamientos generales para la formación docente comunes a los profesorados universitarios" que posteriormente fueron aprobados por el Consejo Interuniversitario Nacional (Res. CE No 787/12) y por el Consejo Universitario (CU).

El mencionado documento señala como eje del Campo en la Formación en la Práctica Profesional a los "Procesos de análisis, intervención, y reflexión reconstrucción de prácticas docentes en contextos macro, meso y micro educativos".

Nuestra Facultad trabaja cada año a fin de consolidar este campo, explicitar las concepciones de práctica que subyacen así como analizar las epistemologías que se construyen. Pensamos la formación como un proceso permanente y continuo que abarca la trayectoria formativa de quienes en ella participan, donde teoría y práctica se vertebran recuperando el valor formativo de ambas en la acción de educar y educarse.

Compartimos en esta editorial propuestas de este campo que se han llevado adelante este año:

- Actividades que ponen en contacto a los/as estudiantes con distintas organizaciones, escolares, comunitarias y/o gubernamentales. Es una instancia de formación en terreno, que permite contrastar en forma integradora, teoría brindada en las asigna- 
turas con empiria, y conectarse desde los primeros años de formación con necesidades de la comunidad en la que deben intervenir, participar y actuar.

- Actividades en relación a la observación y análisis de conmemoraciones, actos escolares, como recurso para indagar en las prácticas docentes, las políticas de memoria y las representaciones vinculadas con las distintas asignaturas que propusieron trabajar con la Historia del Tiempo Presente, la Literatura Argentina en escuelas secundarias de la ciudad.

- Actividades que se desarrollan en las escuelas a modo de talleres donde se ponen en diálogo los saberes específicos disciplinares, la propia biografía, el contexto escolar y la intervención de los/as estudiantes univeristarios/as. Se suman a visitas a instituciones y diálogo con estudiantes y profesoras/es de las escuelas.

- Actividades con profesoras/es de escuelas secundarias con el propósito de vincular las asignaturas universitarias con el curriculum oficial y los espacios curriculares de la escuela. Se analizan las cercanías y distancias, las modalidades de vinculación, la finalidad educativa de secundario y universidad, las diferencias que se advierten según sea le título de grado de las/os profesores que cubren esos espacios curriculares.

- Actividades en relación al análisis de los diseños curriculares para el Secundario. Vinculado con ello la contrastación con libros de texto de distintas editoriales, destinados al nivel secundario, analizando decisiones epistemológicas, políticas, didácticas y curriculares ofrecidas a fin de triangular curriculum prescripto, curriculum editado y curriculum de formación.

- Actividades que favorecen la potencia didáctica de los mediadores simbólicos: relatos, cuentos, novelas, noticias de diarios, entrevistas, cine, diferentes tipos de textos, de distintos ámbitos; conformación de corpus de obras relevantes, y preparación de materiales a través del diseño de clase, de consignas, de actividades de consolidación con ellos para llevar directamente al aula.

- Actividades de vinculación entre asignaturas o grupo de asignaturas a fin de reducir la fragmentación, para llevar adelante una arquitectura de mayor articulación entre saberes, sujetos y prácticas. El trabajo intercátedra de uno o más departamentos favorece la construcción de límites débiles entre el saber disciplinar y pedagógico.

- Diseño de materiales alternativos a los libros de texto que recuperan las voces desoídas en la mirada hegemónica; elaboración de juegos, de textos en español.

- Ayudantías en escuela secundaria acompañando las actividades propuestas por cada docente. Se busca participar institucionalmente del Campo de la Práctica así como comprender las prácticas curriculares, -que en tanto prácticas sociales histórica y políticamente construidas- no pueden ser entendidas sino interpretativa y críticamente.

- Actividades en relación a la organización de los contenidos apuntando a la compresión de conceptos claves en las asignaturas. Diseño de estructuras didácticas, confección de cronologías comparativas, articulación entre saberes de dos asignaturas.

- Actividades de diseños de planificación y elaboración de clases, secuencias didácticas, destinadas a alumnos/as del nivel secundario en las que se incluyen actividades, recortes de contenidos a enseñar, integración con fuentes de análisis y preparación de material audiovisual para trabajar los contenidos.

- Actividades en relación a diseños de investigación y enseñanza, para investigar las propias prácticas y las decisiones profesionales en el aula.

- Actividades en relación a salidas de campo, registros escritos, audiovisuales, digitales, fotográficos para reconocer el espacio, tiempo y cultura escolar.

- Diseño e implementación de secuencias didácticas desarrolladas en las instituciones secundarias y socialización revisando y reflexionando acerca de la práctica.

Aun persisten dificultades / problemáticas para seguir profundizando y/o analizando que se ubican en desestructurar discursos y prácticas instituidas en relación al status epistemológico de teoría y práctica; lugar de lo disciplinar 
y lo pedagógico, de la escuela y de la universidad, lugar de las jerarquías, y el ingreso temprano a la escuela.

Todos los artículos de este volumen se vinculan a este eje: la FORMACIÓN.

Y nos ayudan a pensarla. Diana MAFFÍA ayudando a discutir la formación de ese sujeto hegemónico que naturaliza su posición y legitima una jerarquía y sus argumentos a favor de la diferencia, de la otra y de un contrato moral que se funda en el reconocimiento y la acción colectiva.

Silvia SIDERAC analiza una formación que crea en el Campo de las Prácticas; un programa para curricularizar la formación en derechos humanos y la creación de una cátedra extracurricular para acompañar un proceso similar en problemáticas de educación sexual integral a fin de ofrecer una formación integral.

Emilio Polo FRIZ y ROMERO invitan a pensar la brecha existente entre el explosivo crecimiento de la matrícula de ingresantes y el escaso número de graduados. En ese contexto analizan los procesos de desgranamiento y lentificación en el recorrido de los trayectos curriculares que aumentan la distancia entre la duración teórica y la duración real de las carreras universitarias.

GÓMEZ propone analizar las características de los jóvenes que transitan la primera etapa universitaria, teniendo como objetivo el dar cuenta de algunas referencias psicosociales de los mismos, a los fines de identificar aspectos vinculados a las vivencias en el nuevo trayecto y las formas de acción en torno a la construcción de su lugar en estos nuevos espacios institucionales.

MACHADO analiza el discursos de los estudiantes que valoran la escuela. Se propone discutir, tensionar aquellos discursos que pesan sobre los/as jóvenes, sus escuelas y sus barrios, en especial sobre quienes viven en contextos de extrema pobreza urbana, recuperando sus voces, aquello que dicen sobre su escolaridad, su futuro, sus deseos y temores.

Cerramos con la narrativa de Ricardo NERVI, maestro, que ilustra cada volumen con su pensamiento acerca de la formación y de la docencia.

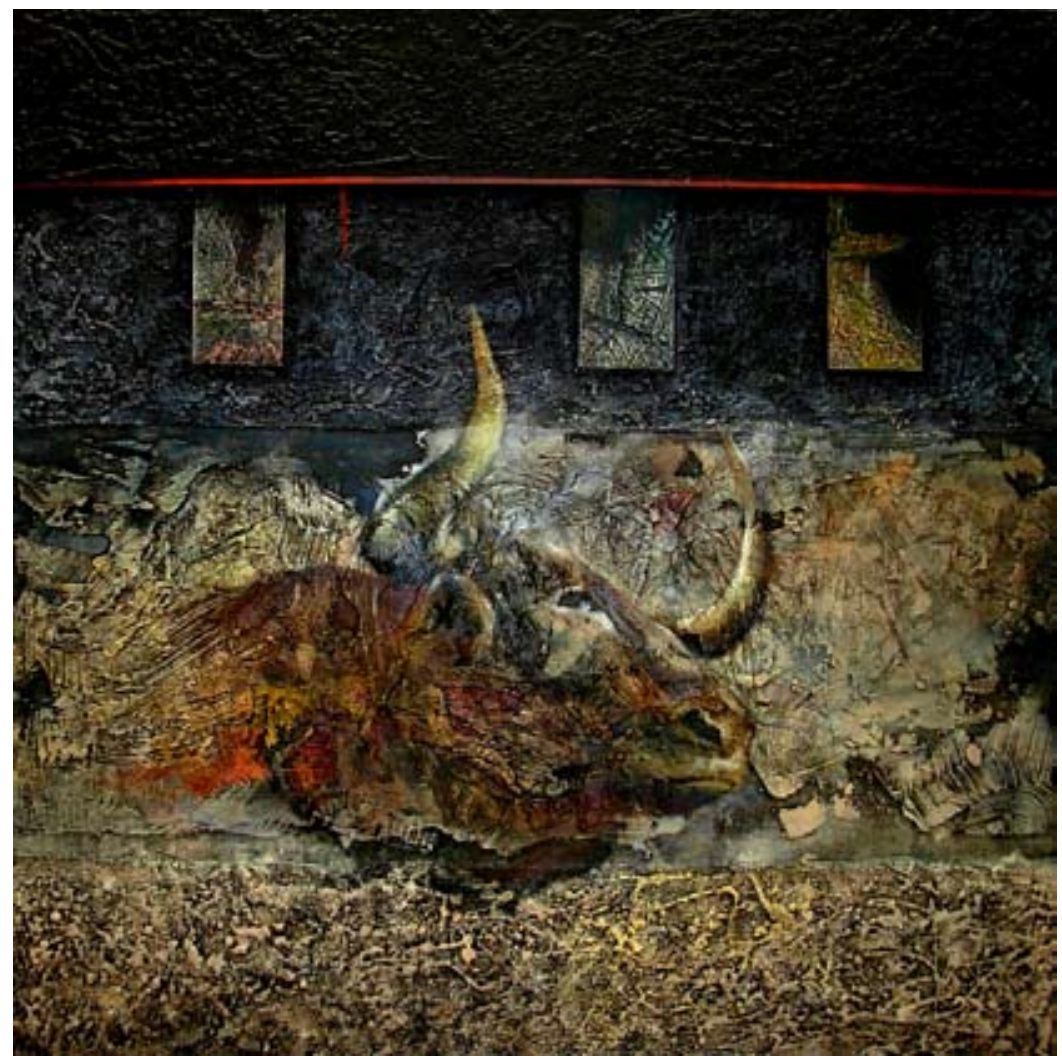

"Behigorri", acrílico. Marta Arangoa 